\title{
Paper No 3.1: A Contact Lens With Built-In Display: Science Fiction or Not?
}

\author{
Herbert De Smet, Jelle De Smet, Dieter Cuypers, Ching-Pao Weng, and Pankaj Joshi \\ Centre for Microsystems Technology (CMST), IMEC and Ghent University, Technologiepark 914 - \\ 9052, Gent, Belgium
}

\begin{abstract}
Recent progress in microsystems integration technology such as ultra-thin chip packaging, stretchable interconnections, thin-film batteries and organic photovoltaics makes it feasible to incorporate various electronic components and transducers in extremely confined spaces and inside flexible or conformable objects. Can this ultimately lead to a genuine display in a contact lens? The major outstanding issues are reviewed.
\end{abstract}

\section{Introduction}

In recent years, advanced integration technologies such as ultrathin chip packaging [1] and stretchable interconnections [2] have become a reality. These technologies make it possible to integrate chips and other electronic components, sensors and transducers into thin organic foils, leading to a flexible and sometimes even stretchable system. The potential of these advanced technologies is tremendous. Intelligent clothing, wearable ECG (electro cardiogram) systems [3] and implantable electronics [4] are just some of the many possible applications of this enabling technology.

Another family of applications one could think of is commonly referred to as 'smart contact lenses'. A contact lens is composed of a transparent and flexible or at least conformable material shaped in such a way that it improves the vision of the person whose eye it is installed upon. The shape of a corneal contact lens is approximately that of a thin circular foil that is spherically curved so that it fits the cornea of the eye. A scleral contact lens is somewhat larger and has a more complex curvature so that it rests on the sclera (white part of the eye) while bridging the cornea, but it is still approximately a curved circular foil.

Since the technologies are available to incorporate electronic components and transducers into a thin foil, one could contemplate integrating some degree of intelligence into a contact lens, as depicted in Figure 1. Some possible applications of such a system are:

- Medical sensors, such as e.g., a permanent eye pressure sensor to allow early diagnosis and prevention of glaucoma, the no. 2 cause of irreparable blindness.

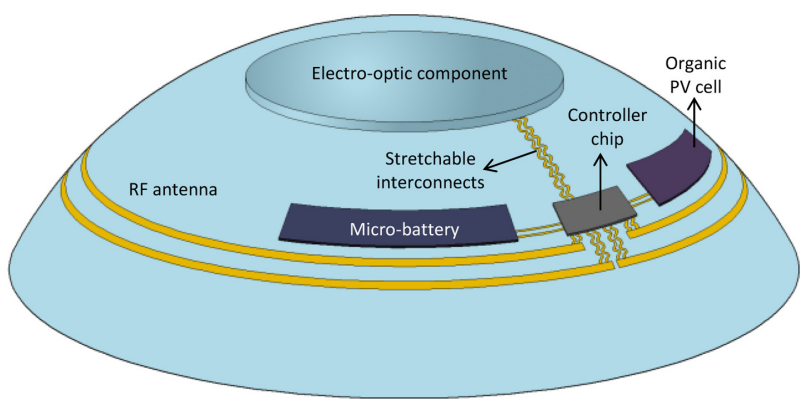

Figure 1. Concept of a smart contact lens.
- Electronically tunable eye sight correction, counteracting the effects of presbyopia (reduced accommodation ability).

- A built-in information display system showing symbology or images superimposed on the normal viewing.

This paper addresses the outstanding issues for the realization of the built-in display application.

\section{Prior Work}

One "smart contact lens" application that has already been demonstrated [5] is an eye pressure sensor, comprising the actual (strain gauge based) sensor, a signal conditioner chip, and a wireless communication and powering circuit. The use of this system requires the wearing of a head-mounted patch surrounding the eye that wirelessly powers the system and reads out the measurement data. While being useful in a medical context, this application illustrates that a more appropriate power supply is necessary to allow autonomous use.

A group of the University of Washington has demonstrated the incorporation of micro-LEDs onto a contact lens. These LEDS are radio frequency (RF) powered from a distance of $10 \mathrm{~cm} \mathrm{[6].}$

Recently, we have reported [7] that it is possible to produce a spherically curved guest-host liquid crystal display (GH-LCD) with curvature radiuses and total thickness compatible with contact lens shapes (Figure 2). The process flow is shown in Figure 3 . The power consumption of this display module is extremely low and it is to be expected that a built-in microbattery can power such a display.

\section{Outstanding Issues}

\subsection{Image Formation}

A human eye cannot focus on a distance closer than about 250 $\mathrm{mm}$. Therefore, an image generated inside the contact lens will not lead to a sharp image on the retina of its wearer. Nevertheless, an integrated multipixel LCD cell can still be useful for other applications, such as an artificial iris or adaptive vision correction. This will be reported elsewhere. To actually display information visible to the wearer, an auxiliary optical system is needed. One conceivable approach is to integrate a distributed micro-LED microprojector inside the lens. In this concept, every micro-LED has its own microlens that collimates the light and

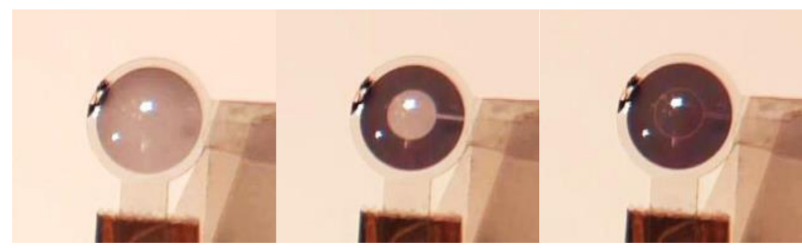

Figure 2. Spherically curved guest-host LCD compatible with contact lens shape. 
a.

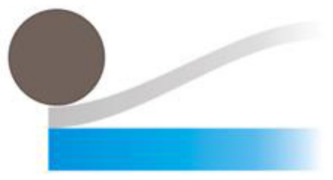

b.

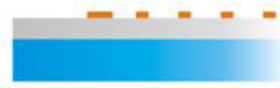

c.

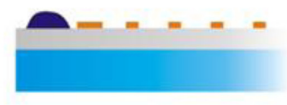

d.

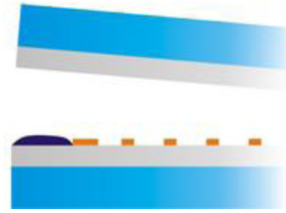

e.

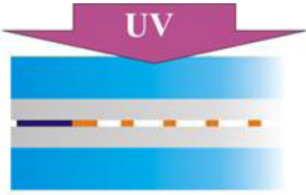

f.
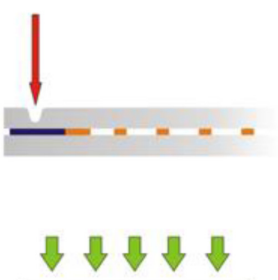

g.

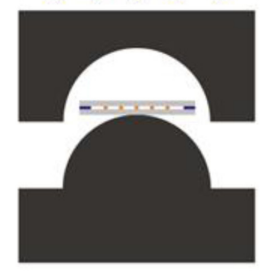

h.

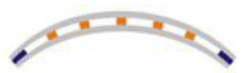

Figure 3. Process flow of the curved GH-LCD cell. (a) PET films laminated onto rigid carrier; (b) photolithographic seal and spacer pattern; (c) glue dispensed; (d) top layers deposited; (e) stack pressed together and UV cured; (f) removal of carriers and laser cutting of the lenses; (g) molding; and (h) resulting cell, ready to be filled and sealed [7].

projects it onto the retina of the eye. Ray-tracing simulations performed in Zemax, using a realistic eye model and the HuyghensFresnel approximation to take into account diffraction effects, show that the limited available clearance between the microLEDs and the microlenses makes it very hard to generate sufficiently sharp light spots on the retina. The best approach found is to space the micro-LEDs at $100 \mu \mathrm{m}$ and integrate a $1-\mu \mathrm{m}$ pinhole in front of every micro-LED. Using lenses with $60 \mu \mathrm{m}$ diameter positioned $100 \mu \mathrm{m}$ away from the pinholes, light spots of $400 \mu \mathrm{m}$ are projected onto the retina (Figure 4). While these spots are considerably smaller than the size of the retina and even smaller than the fovea $(2 \mathrm{~mm})$, it has to be remarked that the actual high-definition region of the fovea (the foveola) is only $0.2 \mathrm{~mm}$ in diameter. It is that region that we use for our sharp vision, reading text, etc. Clearly, the resolution that we can reach with the described optical concept is too low for highresolution symbols or characters to be imaged onto the retina. The optical limitation is mainly attributed to the limited allowed distance between the LEDs and the microlenses, which in its turn is determined by the maximum thickness of the contact lens (preferably less than $150 \mu \mathrm{m}$ ). In principle, the use of holographic components instead of simple microlenses could allow the generation of sharper patterns on the retina, but this requires further research. It remains to be seen how the human visual system will perceive a pattern projected onto the retina by such an

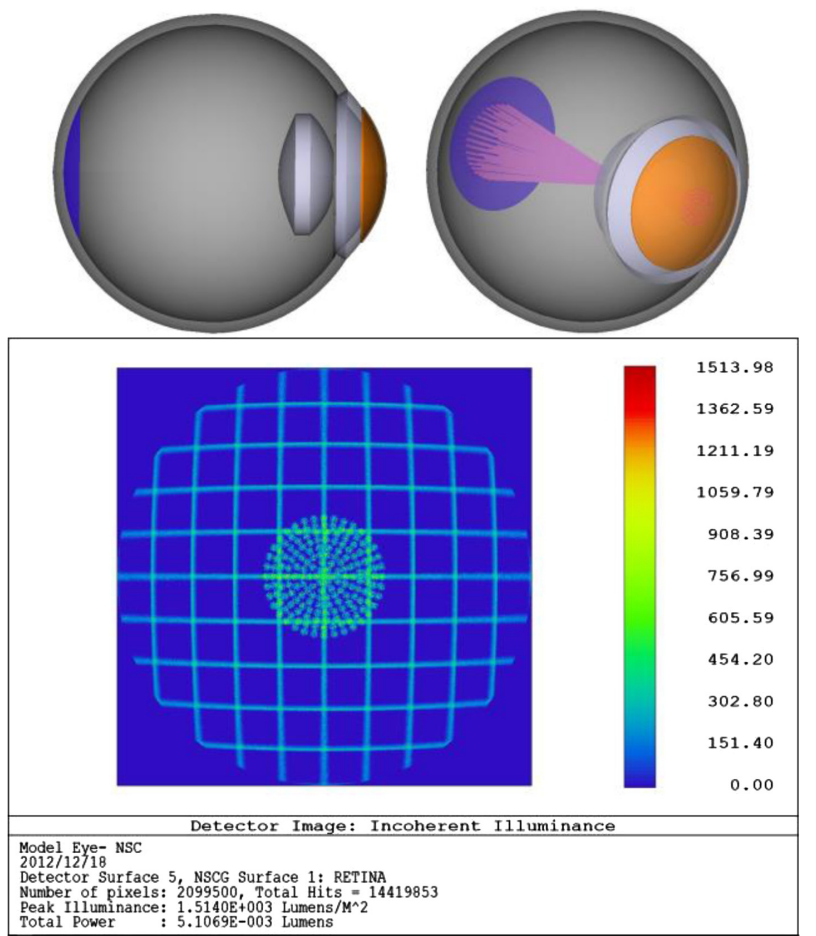

Figure 4. Simulation result of distributed micro-LED projector. Top-left: eye model with contact lens; topright: angled view of projection; bottom: Zemax simulation result of image formed on retina. The microLEDs and hence also the projected spots are arranged in concentric rings.

optical system. In the worst case, only limited information such as intensity and color hue will be perceived. In the best case, it will be possible to display directional information or lowresolution images.

\subsection{Energy Provision}

A system in a contact lens must be completely self-contained, that is, it is not allowed to use wires for the energy supply.

The integration of an energy-harvesting component such as a thin organic photovoltaic (PV) cell is one possible way to supply energy wirelessly. A lot of progress has been made in the organic PV technology and the reported efficiencies have roughly gone up from $3 \%$ to $9 \%$ in the last 5 years [8].

Another technique that is feasible involves radiofrequency (RF) energy transfer using a single- or double-turn loop RF coil integrated into the lens [9]. This technique is well known from RFID applications.

A microbattery can provide autonomy for operation when the energy harvester is not contributing. Thin-film solid-state lithium microbatteries are good candidates for integration into a contact lens as they are very thin (active stack $<10 \mu \mathrm{m}$ ), do not contain liquid electrolytes, and are compatible with a flexible substrate.

In a practical system, the battery could be recharged overnight in a dedicated storage container with lens cleaning fluid and a provision to charge the battery via either light (PV cell) or RF energy transfer (integrated coil). As the lens is not in proximity of the eye during the recharging, high energy transfer rates can 
be used without limits imposed for the safety of the eye. Also, due to the very short distance between the lens and the recharger, the wireless energy transfer can be very efficient.

\subsection{Biocompatibility}

A smart electronic contact lens has to be comfortable and safe to wear before it will be accepted by the public. The applicable regulations are also very strict. Agencies such as the Food and Drug Administration and the Medicines and Health Regulatory Agency require strict adherence to proven safety criteria prior to licensing any drug, implant, or contact lens for human use. The procedure to gain approval is long and involves extensive testing.

In the case of smart contact lenses, the primary concerns are the biocompatibility of the outer material, the reliable and hermetic encapsulation of potentially harmful components, and the overall oxygen permeability of the lens. In a healthy eye, the cornea-which does not have blood vessels-is provided with the necessary nutrients by means of the existing tear film. The necessary oxygen supply is provided from the surrounding air, by diffusion through this tear film. Even in passive contact lenses, the oxygen permeability is a concern, and materials research is still ongoing to further improve this characteristic. Obviously, a smart contact lens containing electronic devices should not block the oxygen supply to the eye.

Conversely, most of the embedded components (LCD cell, organic PV cell, thin-film battery, etc.) are strongly affected by oxygen and have to be encapsulated to prevent oxygen influx. The encapsulation is also necessary to reduce the risk of leakage of hazardous materials toward the lens material and the tear film. Usable biocompatible encapsulation layers are nanolaminate systems produced by atomic layer deposition (ALD) or multilayer systems based on parylene and ALD nanolaminates.

The requirements mentioned in the above two paragraphs are conflicting requirements and have to be reconciled by taking measures to facilitate the permeation of oxygen through the remaining area in between the encapsulated components. The first and most straightforward measure is of course the maximization of the free area between the components. Furthermore, techniques such as fenestrations and microfluidics can be used to aid the flow of oxygen through the lens, but this adds to the complexity of the complete system.

\subsection{Integration and Manufacturability}

As the target shape is a spherically curved thin lens, the active components have to be attached to a carrier substrate that is also spherically curved and thin. This carrier can be flat during the building of the integrated circuit and molded into the curved shape afterward. Wrinkle-free draping of a flat surface over a spherical shape involves stretching, that is, the distance along the surface between two points on said surface can change. This is unlike draping a flat surface over a cylinder or a cone in which the mutual distances along the surface are preserved. As a result, spherical conformability is a far more demanding target than cylindrical or conical conformability.

All components that are to be integrated have to be flexible and sufficiently thin to fit into the target shape. Silicon chips become flexible if they are sufficiently thinned down by back side grinding. However, they do not become stretchable. Therefore, these chips have to be very small or designed in such a way that during the spherical molding they are deformed approximately onto a conical surface. This can be achieved by making slim ringshaped or banana-shaped chips that are concentric with the lens (Figure 5).

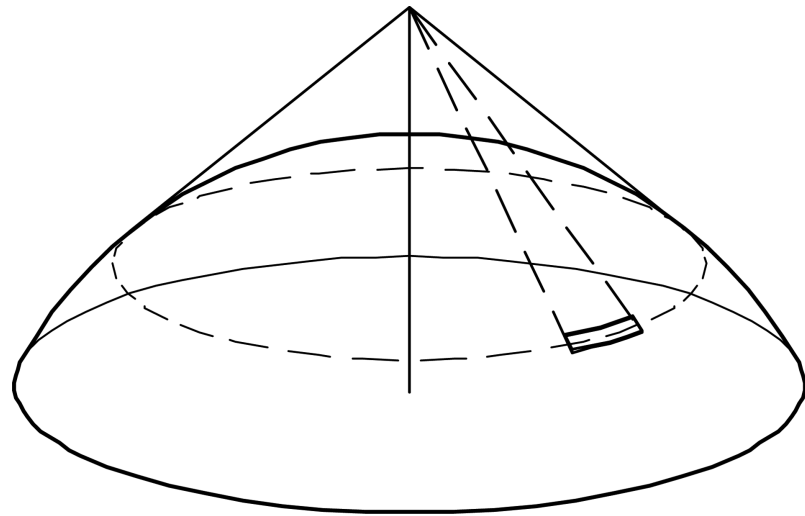

Figure 5. A slim chip oriented along a circle on a spherical surface approximately fits onto the surface of the cone that is tangent to that circle.

The interconnections between the individual components have to be stretchable to accommodate for the spherical molding. This implies that ductile and/or meander-shaped conductors must be used (see Figure 1). Components that cannot be made slim enough have to be ductile in order to withstand the deformation.

For those applications where hard contact lens materials (rigid gas permeable) will be used, the end product of the integration must be "buttons" (flat cylinders) that can be shaped into lenses using the conventional lathing process. The spherically preshaped electronic circuit including all necessary components must be suspended in that button like a fossilized insect in a barn stone.

For applications where soft materials are preferred, a similar approach can be used, followed by a hydration step in order to allow the soft lens material to reach its final state. Alternatively, a cast-molding technique can be used, which immediately polymerizes the lens in the correct shape, but in either case, the electronic circuit including all components must be provided in a form that is compatible with the molding and curing processes.

\subsection{Eye Movements}

Only a very small fraction of the retina of the human eye (the fovea centralis) has a high density of light-sensitive cells, and only the central part of the fovea, the foveola, has a sufficiently high density of cones to allow demanding visual tasks such as reading, The process of acute vision (e.g., when reading), therefore, implies rapid eye movements (saccades) that allows the fovea and the foveola to scan the environment. The brain combines all this information and constructs the perception that the whole environment is sharp.

If it is technically possible to project a sharp image onto the retina, only the part that overlaps with the foveola, and to a lesser extent the fovea, will be perceived in a sharp manner.

As a contact lens moves with the eye, the concept of projected imagery that overlays the normal vision cannot be implemented in a straightforward way. Instead, new concepts are needed that take into count the limited foveola area and compensate for the scanning behavior of the eye.

\subsection{Controller Chip}

At the heart of a smart contact lens is a controller chip. Because of space constraints, it is preferred that this chip takes care of 
both the energy and the data handling. The chip must deal with the energy harvesting from the RF coil and/or the organic PV cell, the battery charging, the interfacing of the integrated sensors, the driving of the display component, and the communication to and from the lens.

If holographic projection is used, this chip is also responsible for the calculation of the holographic patterns that will result in a sharp image on the foveola and the compensation of the rapid eye movements. The design of a compact controller chip that can fulfill all these tasks poses formidable challenges.

\section{Discussion}

In the previous section, some of the major outstanding issues encountered when trying to make a smart contact lens with an integrated information display are described. It is clear that every single one of the listed issues represents a great technological challenge. For some of the issues, it is safe to assume that a viable solution exists, but most of these solutions require significant advances in technology that cannot be made without important additional research efforts. The combination of all existing challenges is no less than formidable and it is clear that a contact lens display is not a short-term development. On the other hand, some alternative, less-challenging applications can be conceived that rely on only about half of the described developments. For example, an artificial iris only requires the development and integration of a thin-film battery, an organic PV cell that acts as the energy harvester and doubles as a light sensor, and a relatively simple controller chip. The light modulator component for this application has already been demonstrated [7] and is available. A similar situation is encountered for the development of a sensor application (e.g., glucose sensor or intraocular pressure sensor), except that the light-modulator component is replaced by the sensor and the controller chip has to be able to store and communicate the measurement data. The major issue for these alternative applications is the biocompatibility issue.

\section{Conclusion}

For the first time, an extensive lineup of the issues faced when trying to fabricate a smart contact lens with integrated display functionality is made. The challenges one faces are formidable, although we believe that solutions can be found for most of them in the longer term. A number of less-challenging applications of this technology platform that can be realized in a shorter term have also been identified.

\section{Acknowledgements}

Part of this work was carried out with sponsorship from the Research Foundation Flanders (FWO), project "Study of liquid crystals dispersed in micro-structured elastic host materials," grant \#G.A047.11N, and from the Agency for Innovation by Science and Technology (IWT), SBO project SECONDOS, grant \#120019.

\section{References}

[1] W. Christiaens, E. Bosman, J. Vanfleteren, "UTCP: A novel polyimide-based ultra-thin chip packaging technology", IEEE Trans. Compon. Packaging Technol., Vol. 33, pp. 754-760, 2010.

[2] A. Jahanshahi, P. Salvo, J. Vanfleteren, "Reliable stretchable gold interconnects in biocompatible elastomers", J. Polym. Sci. Part B: Polym. Phys., Vol. 50, pp. 773-776, 2012.

[3] T. Sterken, J. Vanfleteren, T. Torfs, M. Op de Beeck, F. Bossuyt, C. Van Hoof, "Ultra-thin chip package (UTCP) and stretchable circuit technologies for wearable ECG system", 2011 Annual International Conference of the IEEE Engineering in Medicine and Biology Society (EMBS), pp. 6886-6889, 2011.

[4] A. Jahanshahi, P. Salvo, J. Vanfleteren, "Stretchable biocompatible electronics by embedding electrical circuitry in biocompatible elastomers", Proceedings in 34th Annual International Conference of the Engineering in Medicine and Biology Society, pp. 6007-6010, 2012.

[5] Available from: http://www.sensimed.ch/en/technology/ telemetry.html.

[6] J. Pandey, Y.-T. Liao, A. Lingley, R. Mirjalili, B. Parviz, B. P. Otis, "A fully integrated RF-powered contact lens with a single element display", IEEE Trans. Biomed. Circuits Syst., Vol. 4, pp. 454-461, 2010.

[7] J. De Smet, A. Avci, R. Beernaert, D. Cuypers, H. De Smet, "Design and wrinkling behavior of a contact lens with an integrated liquid crystal light modulator", IEEE J. Display Technol., Vol. 8, pp. 299-305, 2012.

[8] G. Li, R. Zhu, Y. Yang, "Polymer solar cells", Nat. Photon., Vol. 6, pp. 153-161, 2012

[9] Y.-T. Liao, H. Yao, et al., "A 3- $-\mu \mathrm{W}$ CMOS glucose sensor for wireless contact-lens tear glucose monitoring", IEEE J. Solid-State Circuits, Vol. 47, pp. 335-344, 2012. 